\title{
Prevalence, antimicrobial resistance, and molecular characterization of Campylobacter spp. in bulk tank milk and milk filters from US dairies
}

\author{
Laura P. Del Collo, ${ }^{*} †$ Jeffrey S. Karns, ${ }^{*}$ Debabrata Biswas,† Jason E. Lombard,‡ Bradd J. Haley, ${ }^{*}$ \\ R. Camilla Kristensen, $\ddagger$ Christine A. Kopral, $\ddagger$ Charles P. Fossler, $\ddagger$ and Jo Ann S. Van Kessel ${ }^{* 1}$ \\ *Environmental Microbial and Food Safety Laboratory, USDA-Agricultural Research Service, Beltsville, MD 20705 \\ †Department of Animal and Avian Sciences, University of Maryland, College Park 20742 \\ $\ddagger$ Center for Epidemiology and Animal Health, USDA-Animal and Plant Health Inspection Service, Veterinary Services, Fort Collins, CO 80526
}

\section{ABSTRACT}

Campylobacter spp. are frequently isolated from dairy cows as commensal organisms. Sporadic Campylobacter infections in humans in the United States are generally attributed to poultry, but outbreaks are also commonly associated with dairy products, particularly unpasteurized or raw milk. Bulk tank milk samples and milk filters from US dairy operations were collected during the National Animal Health Monitoring System Dairy 2014 study and analyzed using real-time PCR and traditional culture techniques for the presence of thermophilic Campylobacter species. The weighted prevalence of operations from which we detected Campylobacter spp. in either bulk tank milk or milk filters was $24.9 \%$. We detected Campylobacter spp. in a higher percentage of operations with 100-499 cows (42.8\%) and 500 or more cows $(47.5 \%)$ than in operations with 30-99 cows (6.5\%). Campylobacter spp. were also more frequently detected in operations in the west than the east (45.9 and $22.6 \%$, respectively). We isolated Campylobacter spp. from approximately half of PCR-positive samples, representing $12.5 \%$ (weighted prevalence) of operations. The majority $(91.8 \%)$ of isolates were $C$. jejuni, but $C$. lari and $C$. coli were also isolated. We detected resistance to tetracycline in $68.4 \%$ of $C$. jejuni isolates, and resistance to ciprofloxacin and nalidixic acid in $13.2 \%$ of C. jejuni isolates. Based on pulsed-field gel electrophoresis, we found that dairy-associated C. je$j u n i$ were genotypically diverse, although clonal strains were isolated from different geographic regions. These results suggest that bulk tank milk can be contaminated with pathogenic Campylobacter spp., and that the consumption of unpasteurized or raw milk presents a potential human health risk.

Received September 30, 2016.

Accepted December 17, 2016.

${ }^{1}$ Corresponding author: joann.vankessel@ars.usda.gov
Key words: Campylobacter, bulk tank milk, milk filter

\section{INTRODUCTION}

Campylobacter spp. are microaerophilic, spiralshaped, gram-negative bacteria that are a leading cause of bacterial diarrhea in humans in the United States, accounting for an estimated 1.3 million cases and 120 deaths annually (CDC, 2016). Campylobacteriosis is usually self-limiting, with symptoms such as mild diarrhea and cramping; however, severe cases may require antimicrobial treatment. Post-infection sequelae such as Guillain-Barré syndrome may cause lifelong health problems. According to preliminary 2015 data published by the Centers for Disease Control and Prevention (CDC), Campylobacter spp. infections in humans increased by $9 \%$ from 2006 to 2015 (CDC, 2016). The most common cause of campylobacteriosis in humans is Campylobacter jejuni, but other thermophilic species such as Campylobacter coli and Campylobacter lari have also been known to cause disease (Taylor et al., 2013; CDC, 2016).

Although sporadic Campylobacter spp. infections are most commonly associated with poultry, outbreaks have also been attributed to the consumption of other foods, particularly unpasteurized or raw milk. Based on a review of CDC data from 1997 to 2008, 28\% of foodborne Campylobacter outbreaks (defined as 2 or more affected people) were due to the consumption of contaminated raw milk products, compared with $11 \%$ due to the consumption of poultry (Taylor et al., 2013). Langer et al. (2012) and Mungai et al. (2015) analyzed milk-related outbreak data from 1993 to 2006 and 2007 to 2012, respectively, and found that the majority of outbreaks associated with unpasteurized milk were due to Campylobacter contamination. Furthermore, in a recent analysis of Campylobacter spp. isolated from human and animal sources, Tyson et al. (2016) discovered that isolates from human clinical samples more frequently matched isolates from dairy cattle $(65.0 \%)$ than isolates from poultry sources (49.5\%). Therefore, 
dairy cattle and dairy products, particularly unpasteurized milk, should be considered an important source of Campylobacter spp. infection.

Dairy cows are known reservoirs of Campylobacter spp., and several surveys have determined the prevalence of Campylobacter spp. on dairy farms. According to National Animal Health Monitoring System (NAHMS) studies in 1996, 2002, and 2007, Campylobacter spp., primarily C. jejuni, were detected in the feces of cows on $90-100 \%$ of US dairy operations (USDA-APHIS, 2011). The within-herd prevalence of most operations was greater than $10 \%$. Dairy cows have been described as intermittent shedders; however, cows may also shed C. jejuni consistently or sporadically in high concentrations (Häkkinen and Hänninen, 2009; Rapp et al., 2012). Campylobacter spp. have also been detected in bulk tank milk (BTM; Halbert et al., 2006). Although Campylobacter spp. have occasionally been implicated in cases of mastitis (Morgan et al., 1985; Orr et al., 1995; Bianchini et al., 2014) and can be excreted in the milk of infected cows, this is an uncommon occurrence, and the presence of Campylobacter spp. in BTM is usually due to fecal contamination (Oliver et al., 2005). Therefore, even with good animal hygiene and strict milking protocols, some level of contamination in the bulk tank by asymptomatic animals is inevitable.

Pasteurization is a highly effective tool for managing BTM contamination; however, raw milk can be legally distributed in some manner in more than half of US states (AAP, 2014; NCSL, 2016). Although dairy animals are common carriers of Campylobacter spp. and fecal shedding can lead to BTM contamination, no national surveys have been conducted on the prevalence of Campylobacter spp. in BTM in the United States. Sampling milk filters in addition to BTM can also improve the ability to detect pathogens in BTM (Van Kessel et al., 2008; Latorre et al., 2011; Van Kessel et al., 2011). The objective of this study was to determine the prevalence of Campylobacter spp. in BTM and milk filters from US dairy operations from samples collected during the NAHMS Dairy 2014 study.

\section{MATERIALS AND METHODS}

To identify operations that would participate in the NAHMS Dairy 2014 study, a stratified random sample of dairy operations was selected from the USDA National Agricultural Statistics Service list frame. The survey design was a stratified random sample with unequal selection probabilities to ensure the inclusion of dairy operations with 500 or more cows and organic operations. Operations were chosen from each of 17 dairy states in 2 regions of the country (west: California, Colorado, Idaho, Texas, and Washington; east: In- diana, Iowa, Kentucky, Michigan, Minnesota, Missouri, New York, Ohio, Pennsylvania, Vermont, Virginia, and Wisconsin). These states represent $80.5 \%$ of dairy herds and $81.3 \%$ of dairy cows in the United States. Operations were classified into 3 herd size categories based on the number of cows: small (30-99 cows), medium (100-499 cows), and large (500 or more cows).

Producers $(\mathrm{n}=3,500)$ that reported 1 or more milk cows in their inventory on January 1, 2014, were selected for phase I of the NAHMS Dairy 2014 study. During this phase, National Agricultural Statistics Service enumerators administered a general management questionnaire to participating producers ( $\mathrm{n}=$ 1,191). In phase II, federal and state veterinary medical officers or animal health technicians administered an additional questionnaire to operations with 30 or more milk cows on January 1, 2014, that had participated in phase I and agreed to continue participating in the study $(\mathrm{n}=265)$.

Samples from BTM and milk filters were collected from March to July 2014. Trained staff aseptically collected BTM (50-150 mL) and milk filters from participating operations. Sample collectors were instructed to ensure that the BTM and milk filter samples represented at least 1 complete milking cycle of the herd. Thus, if the herd had more than 1 bulk tank, then each bulk tank was sampled, and all milk filters used during a milking cycle were collected. Samples were shipped overnight on ice to the USDA-Agricultural Research Service in Beltsville, Maryland.

For enrichment of Campylobacter spp. from BTM, multiple tubes of milk from an operation were first combined and mixed, and then a $25 \mathrm{~mL}$ aliquot was centrifuged at 20,000 $\times g$ for $35 \mathrm{~min}$ at $8^{\circ} \mathrm{C}$ (Figure 1). The supernatant was discarded, and the pellet was resuspended in $45 \mathrm{~mL}$ of Bolton Broth (Oxoid, Basingstoke, UK) with 5\% Laked Horse Blood (Lampire, Pipersville, PA) and a Campylobacter selective supplement that contained cefoperazone, cycloheximide, trimethoprim, and vancomycin (Dalynn, Calgary, AB, Canada). For enrichment of Campylobacter spp. from milk filters, the filters were cut into small pieces and placed in filtered stomacher bags, to which $1 \%$ buffered peptone water (1:1, wt/wt) was added, as previously described by Van Kessel et al. (2011). The bags were pummeled in an automatic bag mixer for 2 min, repositioned, and then pummeled for an additional $2 \mathrm{~min}$. Then, $5 \mathrm{~mL}$ of filtrate was added to $40 \mathrm{~mL}$ of Bolton Broth with 5\% Laked Horse Blood and Campylobacter selective supplement.

For all samples, enrichment tubes were incubated at $37^{\circ} \mathrm{C}$ for $48 \mathrm{~h}$ in a $10 \% \mathrm{CO}_{2}$ incubator with loosened caps. After incubation, $2 \mathrm{~mL}$ of enrichment broth were centrifuged $(12,000 \times g)$. The supernatants were 
discarded, and the bacterial pellets were suspended in $0.5 \mathrm{~mL}$ of $1 \times$ freezer medium (Schleif and Wensink, 1981 ) and stored at $-80^{\circ} \mathrm{C}$. Additionally, $1.5 \mathrm{~mL}$ of enrichment broth was centrifuged $(12,000 \times g)$, and nucleic acids were extracted from the bacterial pellets using $200 \mu \mathrm{L}$ of a commercially prepared extraction medium (Mericon DNA Bacteria Kit Fast Lysis Buffer; Qiagen, Hilden, Germany). The DNA preparations were analyzed by real-time PCR for the presence of C. jejuni, C. coli, or C. lari using a commercial kit (Mericon Campylobacter triple kit; Qiagen) following the manufacturer's instructions. Reactions were run on an Agilent Mx3005P qPCR system (Agilent Technologies, Santa Clara, CA) following the kit manufacturer's cycling protocol. Samples with a cycle threshold of fewer than 40 cycles were considered positive.

After incubation, $30 \mu \mathrm{L}$ of enrichment broth was also streaked onto Campy Blood-Free Selective Medium (Neogen, Lansing, MI), which was supplemented with cefoperazone (Neogen) and incubated at $42^{\circ} \mathrm{C}$ for $48 \mathrm{~h}$ in a microaerophilic environment $\left(10 \% \mathrm{CO}_{2}, 5 \% \mathrm{O}_{2}\right.$, $85 \% \mathrm{~N}_{2}$ ) using an Anoxomat canister system (Mart Microbiology, Norwood, MA). Suspected Campylobacter colonies (2 to 5 ) were transferred from each plate to a panel of agar plates that included Campy Blood-Free

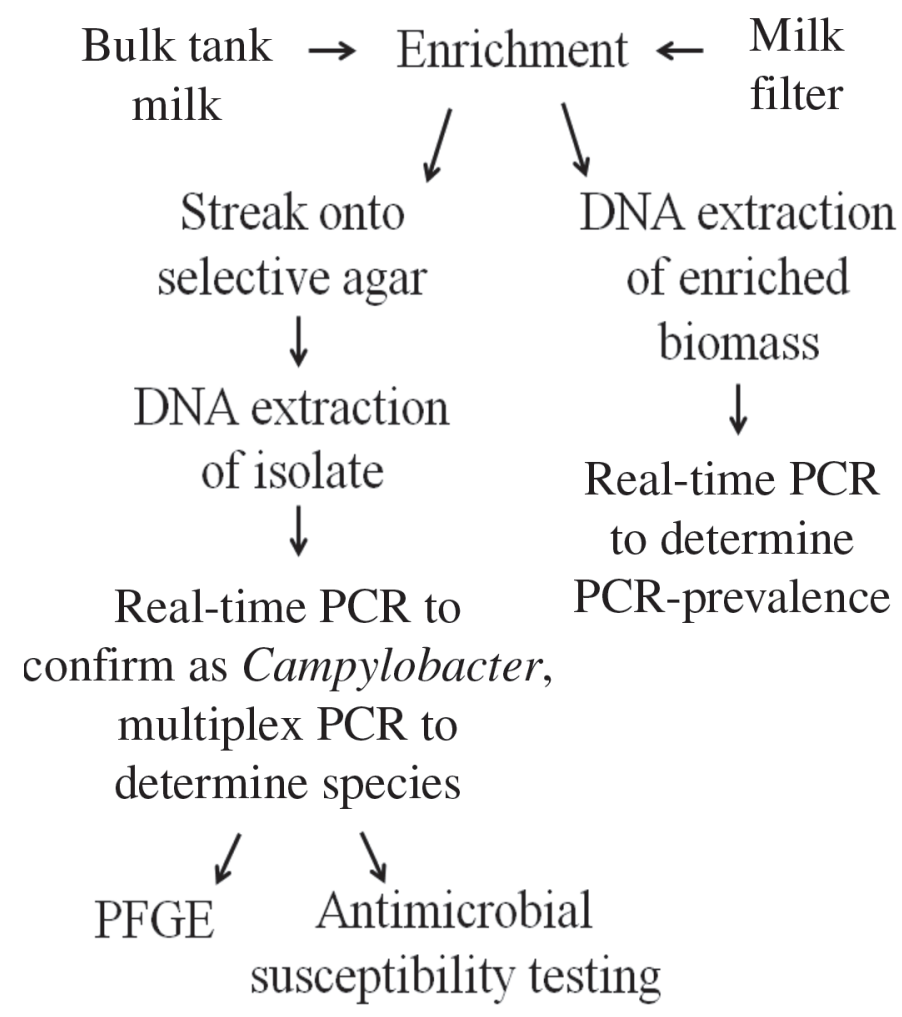

Figure 1. Schematic of bulk tank milk and milk filter sample and isolate processing. $\mathrm{PFGE}=$ pulsed-field gel electrophoresis.
Selective Medium, Columbia Agar with 5\% Sheep Blood (BD, Sparks, MD), Brilliance CampyCount (Remel, Lenexa, KS), C. jejuni/C. coli Chromogenic Plating Medium (R\&F Laboratories, Downers Grove, IL), and Mueller Hinton (Neogen) agar plates. Then, DNA was extracted from 1 phenotypically unique isolate from each sample using an InstaGene Matrix suspension (Bio-Rad, Hercules, CA) following the manufacturer's instructions. Presumptive Campylobacter spp. isolates were confirmed as such using a commercial real-time PCR kit (Mericon Campylobacter triple kit; Qiagen). All isolates were also preserved in $0.5 \mathrm{~mL}$ of a freezer medium consisting of $80 \%$ Bolton Broth, $15 \%$ glycerol, and $5 \%$ Laked Horse Blood, and stored at $-80^{\circ} \mathrm{C}$.

The species of confirmed Campylobacter isolates was determined by multiplex PCR as described by Wang et al. (2002) with modifications. Each reaction mixture $(26 \mu \mathrm{L})$ contained $240 \mu \mathrm{M}$ of a total deoxynucleoside triphosphate mixture (ThermoFisher, Lenexa, KS); $2.5 \mu \mathrm{L}$ of $10 \times$ reaction buffer II; $4 \mathrm{mM} \mathrm{MgCl}_{2} ; 1.5$ U of AmpliTaq Gold polymerase (Applied Biosystems, Foster City, CA); $0.6 \mu M$ each of forward and reverse C. jejuni and C. lari primers; $1 \mu M$ each C. coli and Campylobacter fetus primers; $2 \mu M$ Campylobacter upsaliensis primers; $0.2 \mu M$ 23S rRNA primers; $1.0 \mu \mathrm{L}$ of InstaGene extract; and molecular biology-grade water. The reaction conditions were as follows: an initial incubation at $95^{\circ} \mathrm{C}$ for $10 \mathrm{~min}$, followed by 30 cycles of $95^{\circ} \mathrm{C}$ for $30 \mathrm{~s}, 59^{\circ} \mathrm{C}$ for $30 \mathrm{~s}$, and $72^{\circ} \mathrm{C}$ for $30 \mathrm{~s}$, with a final extension step of $72^{\circ} \mathrm{C}$ for $7 \mathrm{~min}$. The PCR products were separated on a $1.5 \%$ agarose gel in $1 \times$ Tris-borate-EDTA buffer (Invitrogen, Grand Island, NY) containing $0.5 \mu \mathrm{g} / \mathrm{mL}$ ethidium bromide (Bio-Rad) and visualized under UV light. Positive controls were $C$. jejuni NCTC 81116, C. coli ATCC 33559, C. lari 43675, C. fetus ssp. fetus ATCC 33246, and C. upsaliensis ATCC 43954.

Resistance to a panel of 9 antimicrobials used by the National Antimicrobial Resistance Monitoring System (NARMS; azithromycin, ciprofloxacin, erythromycin, gentamicin, tetracycline, florfenicol, nalidixic acid, telithromycin, and clindamycin) was assessed using an automated microdilution procedure (Sensititre; ThermoFisher). Cultures were streaked onto Columbia Agar with $5 \%$ Sheep Blood (BD) and incubated at $42^{\circ}$ for $48 \mathrm{~h}$; biomass from these plates was used for testing. We used C. jejuni ATCC 33560 as a susceptible quality control strain. Antimicrobial MIC were interpreted based on the epidemiological cutoff values used by the NARMS program (Table 1). The MIC that inhibited $50 \%\left(\mathrm{MIC}_{50}\right)$ and $90 \%\left(\mathrm{MIC}_{90}\right)$ of isolates were also calculated (Schwarz, et al., 2010).

The Campylobacter spp. isolates were characterized by pulsed-field gel electrophoresis (PFGE) following the standardized PulseNet C. jejuni protocol (Ribot 
Table 1. Minimum inhibitory concentrations $(\mu \mathrm{g} / \mathrm{mL})$ used to determine resistance of Campylobacter spp. ${ }^{1}$

\begin{tabular}{lcc}
\hline Antimicrobial & C. jejuni/C. lari $^{2}$ & C. coli \\
\hline Azithromycin & $\geq 0.5$ & $\geq 1$ \\
Ciprofloxacin & $\geq 1$ & $\geq 1$ \\
Erythromycin & $\geq 8$ & $\geq 16$ \\
Tetracycline & $\geq 2$ & $\geq 4$ \\
Florfenicol & $\geq 8$ & $\geq 8$ \\
Nalidixic acid & $\geq 32$ & $\geq 32$ \\
Telithromycin & $\geq 8$ & $\geq 8$ \\
Clindamycin & $\geq 1$ & $\geq 2$ \\
Gentamicin & $\geq 4$ & $\geq 4$ \\
\hline
\end{tabular}

${ }^{1}$ Based on epidemiological cutoff values established by the European Committee on Antimicrobial Susceptibility Testing (www.eucast.org). ${ }^{2}$ No specific MIC for C. lari.

et al., 2001) with a few modifications. Cultures were streaked onto Columbia Agar with 5\% Sheep Blood (BD) and incubated at $42^{\circ}$ for $48 \mathrm{~h}$; biomass from these plates was used for agarose plug preparation. Plug slices were incubated overnight with $40 \mathrm{U}$ SmaI (Roche Diagnostics, Mannheim, Germany). The size standard was XbaI-digested Salmonella enterica serotype Braenderup H9812. Thiourea $(50 \mu M)$ was added to both the gel and the electrophoresis buffer. Gels composed of $1 \%$ SeaKem Gold agarose (Lonza, Allendale, NJ) in Tris-borate-EDTA (Sigma, St. Louis, MO) were run on Chef-DR II or Chef-DR III systems (Bio-Rad) for 18.5 $\mathrm{h}$. The gels were stained with $1 \mu \mathrm{g} / \mathrm{mL}$ ethidium bromide (Bio-Rad) and destained with 4 washings. Images of the restriction digest patterns were obtained using a
ChemiDoc XRS gel documentation system (Bio-Rad). Bands were assigned manually and analyzed using BioNumerics software (Applied Maths, Austin, TX). Dendrograms were derived using the unweighted pair group method with arithmetic average cluster analysis, a band position tolerance of $1.0 \%$, and optimization of $0.5 \%$.

The prevalence (\%) of Campylobacter spp. at the operation level was statistically weighted to provide national prevalence estimates. The weighted prevalence (wp) was calculated using statistical software (SUDAAN, release 11.0.1, 2013; Research Triangle Institute, Research Triangle Park, NC), which accounted for the operations' probability of selection and adjusted for non-response. Selection weights and non-response adjustments were based on state and herd size. Estimates of the percentage of positive samples were conducted using SAS version 9.4 (SAS Institute Inc., Cary, NC).

\section{RESULTS}

A total of 234 BTM and 231 milk filter samples were collected from 234 operations in 17 states between March and July 2014 (Table 2). Paired BTM and filter samples were received from 231 dairy operations and BTM only from 3 operations. The number of filters collected from individual operations ranged from 1 to 20 . When necessary, filters from the same operation were processed in multiple enrichments $(\leq 6$ filters per en-

Table 2. State of origin and summary of bulk tank milk (BTM) samples and milk filter samples received from March to July 2014

\begin{tabular}{|c|c|c|c|c|c|}
\hline \multirow[b]{2}{*}{ State } & \multirow{2}{*}{$\begin{array}{c}\text { No. of } \\
\text { operations }\end{array}$} & \multicolumn{4}{|c|}{ Sample counts (no.) } \\
\hline & & BTM & Filter & Pairs $^{1}$ & BTM, no filters ${ }^{2}$ \\
\hline California & 22 & 22 & 22 & 22 & 0 \\
\hline Colorado & 5 & 5 & 5 & 5 & 0 \\
\hline Idaho & 6 & 6 & 6 & 6 & 0 \\
\hline Indiana & 3 & 3 & 3 & 3 & 0 \\
\hline Iowa & 12 & 12 & 12 & 12 & 0 \\
\hline Kentucky & 1 & 1 & 1 & 1 & 0 \\
\hline Michigan & 18 & 18 & 17 & 17 & 1 \\
\hline Minnesota & 24 & 24 & 24 & 24 & 0 \\
\hline Missouri & 2 & 2 & 2 & 2 & 0 \\
\hline New York & 33 & 33 & 33 & 33 & 0 \\
\hline Ohio & 10 & 10 & 10 & 10 & 0 \\
\hline Pennsylvania & 19 & 19 & 19 & 19 & 0 \\
\hline Texas & 1 & 1 & 1 & 1 & 0 \\
\hline Vermont & 1 & 1 & 1 & 1 & 0 \\
\hline Virginia & 8 & 8 & 7 & 7 & 1 \\
\hline Washington & 11 & 11 & 11 & 11 & 0 \\
\hline Wisconsin & 58 & 58 & 57 & 57 & 1 \\
\hline Total & 234 & 234 & 231 & 231 & 3 \\
\hline
\end{tabular}

${ }^{1}$ Number of operations from which both a BTM sample and a filter sample were available.

${ }^{2}$ Number of operations from which BTM samples were submitted but no corresponding filter sample was available. 
richment); however, results from multiple filter samples from the same operation were combined for analysis.

Based on real-time PCR analysis, the weighted prevalence of operations from which Campylobacter spp. were detected in either the BTM sample or the milk filters was $24.9 \%$ (Table 3 ). Of the 234 BTM and 231 milk filter samples, 27 BTM and 69 filter samples were positive for C. jejuni, C. coli, or C. lari. Real-time PCR cycle thresholds of positive samples ranged from 14.3 to 39.5. Overall, enrichments from 80 operations (24.9\%, wp) were positive for Campylobacter species. Sixteen operations (5.3\%, wp) tested positive for Campylobacter spp. in both the BTM and filter samples. Campylobacter spp. were detected in the filter sample but not the BTM for 53 operations (15.1\%, wp), and in the BTM but not the filter for 9 operations (4.3\%, wp). Campylobacter spp. were detected in 2 milk samples (0.2\%, wp) submitted without accompanying filter samples. Campylobacter spp. were detected in BTM or milk filters in a higher percentage of operations with 100-499 cows and 500 or more cows (42.8 and 47.5\%, respectively) than in operations with 30-99 cows $(6.5 \%$, $P<0.0001)$. Campylobacter spp. were also more frequently detected in operations in the west than in the east (45.9 and $22.6 \%$, respectively; $P=0.021$ ).

Campylobacter spp. were isolated via traditional culture methods from the filter samples of 33 operations and from the BTM samples of 14 operations, representing a total of 43 operations (12.5\%, wp). Isolates were obtained from both the BTM and filter samples of 4 operations (1.2\%, wp). Campylobacter spp. were isolated from only the filter samples from 29 operations $(9.4 \%, \mathrm{wp})$ and from only the BTM samples from 9 operations (1.8\%, wp). Isolates were also obtained from 1 BTM sample $(0.1 \%$, wp) that did not have an accompanying filter sample.

When phenotypically unique isolates $(\mathrm{n}=49)$ from BTM and milk filters from 43 operations were analyzed using multiplex PCR, 45 (91.8\%) were identified as $C$. jejuni, 3 were $C$. lari, and 1 was $C$. coli. One milk filter sample yielded both a $C$. jejuni isolate and a $C$. lari isolate. In preparation for antimicrobial susceptibility testing, 7 of these isolates could not be resuscitated from preservation, and 1 isolate repeatedly failed to grow on the microdilution plate used for susceptibility testing. Therefore, 41 Campylobacter spp. isolates (38 C. jejuni, 2 C. lari, 1 C. coli) were tested for resistance to 9 common antimicrobials (Tables 4 and 5). Of the 38 C. jejuni isolates tested, $26(68.4 \%)$ were resistant to tetracycline, and $5(13.2 \%)$ of those were also resistant to both ciprofloxacin and nalidixic acid. Twelve C. jejuni isolates $(31.6 \%)$ were susceptible to all of the 9 antimicrobials. Both C. lari isolates were resistant to ciprofloxacin and nalidixic acid, but the $C$. coli isolate was susceptible to all 9 antimicrobials.

Of the 49 Campylobacter isolates from BTM and milk filters, 42 were analyzed by PFGE (Figure 2). This included 1 C. jejuni isolate for which antimicrobial susceptibility could not be determined due to a failure to grow on the microdilution plate, but excluded 7 isolates that could not be resuscitated from preservation. When the banding patterns of all isolates were compared, the 2 C. lari isolates and 1 C. coli isolate were distinct, sharing less than $40 \%$ similarity with the $C$. jejuni isolates. The 39 C. jejuni isolates clustered separately from $C$. lari and $C$. coli, but we observed significant diversity within the species, and this diversity extended across geographical regions. The C. jejuni isolates grouped into 4 clades, each sharing at least $50 \%$ similarity between isolates within the clade. Eight groups of 2 isolates and 1 group of 3 isolates were indistinguishable (100\% similarity). Of these 9 groups, 4 represented isolates from the same state, and 5 were from differing (and generally non-neighboring) states. Three indistinguishable isolate groups represented isolates from BTM and milk filter pairs from the same operations (pairs 20, 46, 117). Because filters were processed as multiple enrichments when necessary, 1 operation (pair 117) yielded 2 isolates from the milk filter sample as the result of separate enrichments. Although 1 isolate from 1 filter enrichment was indistinguishable $(100 \%$ similarity) from the isolate from BTM, the isolate from

Table 3. Weighted percent of US dairy operations that were positive for Campylobacter (C. jejuni, C. coli, or C. lari) by real-time PCR and culture in bulk tank milk ( $\mathrm{n}=234$ operations) and milk filters $(\mathrm{n}=231$ operations $)$

\begin{tabular}{|c|c|c|c|c|c|c|}
\hline \multirow[b]{2}{*}{ Sample type and result } & \multicolumn{3}{|c|}{ PCR } & \multicolumn{3}{|c|}{ Culture } \\
\hline & Number & Weighted \% & $\mathrm{SE}$ & Number & Weighted \% & $\mathrm{SE}$ \\
\hline Positive filter & 69 & 20.5 & 3.0 & 33 & 10.6 & 2.3 \\
\hline Positive milk and positive filter & 16 & 5.3 & 1.8 & 4 & 1.2 & 0.9 \\
\hline Negative milk and positive filter & 53 & 15.1 & 2.5 & 29 & 9.4 & 2.1 \\
\hline Positive milk and negative filter & 9 & 4.3 & 1.7 & 9 & 1.8 & 0.7 \\
\hline
\end{tabular}


Table 4. Resistance and resistance profiles of Campylobacter jejuni isolates $(\mathrm{n}=38)$ from bulk tank milk and milk filters from US dairies

\begin{tabular}{|c|c|c|c|c|c|}
\hline Resistance & $\begin{array}{l}\text { MIC range } \\
(\mu \mathrm{g} / \mathrm{mL})\end{array}$ & $\begin{array}{c}\mathrm{MIC}_{50}{ }^{1} \\
(\mu \mathrm{g} / \mathrm{mL})\end{array}$ & $\begin{array}{c}\mathrm{MIC}_{90}{ }^{1} \\
(\mu \mathrm{g} / \mathrm{mL})\end{array}$ & $\begin{array}{l}\text { No. of resistant } \\
\text { isolates }\end{array}$ & $\begin{array}{l}\% \text { of } \\
\text { isolates }\end{array}$ \\
\hline Azithromycin & $0.03-0.12$ & 0.06 & 0.06 & 0 & 0.0 \\
\hline Ciprofloxacin (CIP) & $0.03-16$ & 0.06 & 8 & 5 & 13.2 \\
\hline Clindamycin & $<0.03-0.25$ & 0.12 & 0.25 & 0 & 0.0 \\
\hline Erythromycin & $0.06-1$ & 0.25 & 0.5 & 0 & 0.0 \\
\hline Florfenicol & $0.25-1$ & 1 & 1 & 0 & 0.0 \\
\hline Gentamicin & $0.25-1$ & 0.5 & 1 & 0 & 0.0 \\
\hline Nalidixic acid (NAL) & $\leq 4->64$ & $\leq 4$ & $>64$ & 5 & 13.2 \\
\hline Telithromycin & $0.25-4$ & 0.5 & 1 & 0 & 0.0 \\
\hline Tetracycline (TET) & $\leq 0.06->64$ & $>64$ & $>64$ & 26 & 68.4 \\
\hline Susceptible $^{2}$ & & & & 12 & 31.6 \\
\hline \multicolumn{6}{|l|}{ Resistance profile } \\
\hline TET & & & & 21 & 55.2 \\
\hline CIP-NAL-TET & & & & 5 & 13.2 \\
\hline
\end{tabular}

a second filter enrichment was distinct $(<65 \%$ similarity). A second BTM-milk filter pair from a single operation (pair 196) also yielded 2 unique isolates ( $<40 \%$ similarity). Antimicrobial-resistant phenotypes were dispersed across clades. Of the 9 indistinguishable groups, 7 displayed identical antimicrobial resistance or susceptibility profiles.

\section{DISCUSSION}

Campylobacter spp. are a leading cause of foodborne gastrointestinal illness in the United States, accounting for an estimated 13.3 infections per 100,000 people in 2014 (CDC, 2016). Most illnesses are associated with poultry, but Campylobacter outbreaks are also often attributed to the consumption of raw milk (Taylor et al., 2013). The presence of Campylobacter in raw milk is primarily due to fecal contamination. Dairy cows are known reservoirs of Campylobacter; national surveys have consistently identified Campylobacter spp. on US dairy farms, particularly in fecal samples (USDAAPHIS, 2011). Regional and multi-state surveys have also frequently identified Campylobacter spp. in BTM on US dairy farms (Doyle and Roman, 1982; Lovett et al., 1983; McManus and Lanier, 1987; Rohrbach et al., 1992; Jayarao and Henning, 2001; Jayarao et al., 2006). As part of the NAHMS Dairy 2014 study, thermophilic Campylobacter spp. (C. jejuni, C. lari, C. coli) were detected in BTM or filter samples from approximately one-quarter of a representative sample of dairy operations in the United States. This represents the first national survey to determine Campylobacter prevalence in BTM and milk filters in the United States.

In this study, Campylobacter spp. were detected in the filter samples but not in the BTM from 53 operations $(15.1 \%$, wp). Traditionally, BTM is sampled to assess milk quality and bacterial prevalence, and the purpose of the milk filter is to remove large debris, such as bedding, from the milk before storage in the bulk tank. The filter is not designed to remove bacterial pathogens, but bacterial cells may concentrate on the filter along with large debris. Previous work with $\mathrm{Cam}$ pylobacter spp. and other dairy-associated pathogens has illustrated the value of milk filter sampling as a more sensitive method of detecting bacterial pathogens in milk than BTM sampling alone (Van Kessel et al., 2008; Leone et al., 2010; Latorre et al., 2011; Van Kessel et al., 2011; Giacometti et al., 2012; Serraino et al.,

Table 5. Resistance of Campylobacter lari $(\mathrm{n}=2)$ and Campylobacter coli $(\mathrm{n}=1)$ isolates from bulk tank milk and milk filters from US dairies ${ }^{1}$

\begin{tabular}{|c|c|c|c|}
\hline Species & Resistance & No. of isolates & $\%$ of isolates \\
\hline \multirow[t]{2}{*}{ C. lari } & Ciprofloxacin & 2 & 100.0 \\
\hline & Nalidixic acid & 2 & 100.0 \\
\hline C. coli & Susceptible $^{2}$ & 1 & 100.0 \\
\hline
\end{tabular}

${ }^{1}$ Resistance to azithromycin, ciprofloxacin, erythromycin, tetracycline, florfenicol, nalidixic acid, telithromycin, clindamycin, and gentamicin.

${ }^{2}$ Susceptible to all 9 antimicrobials. 


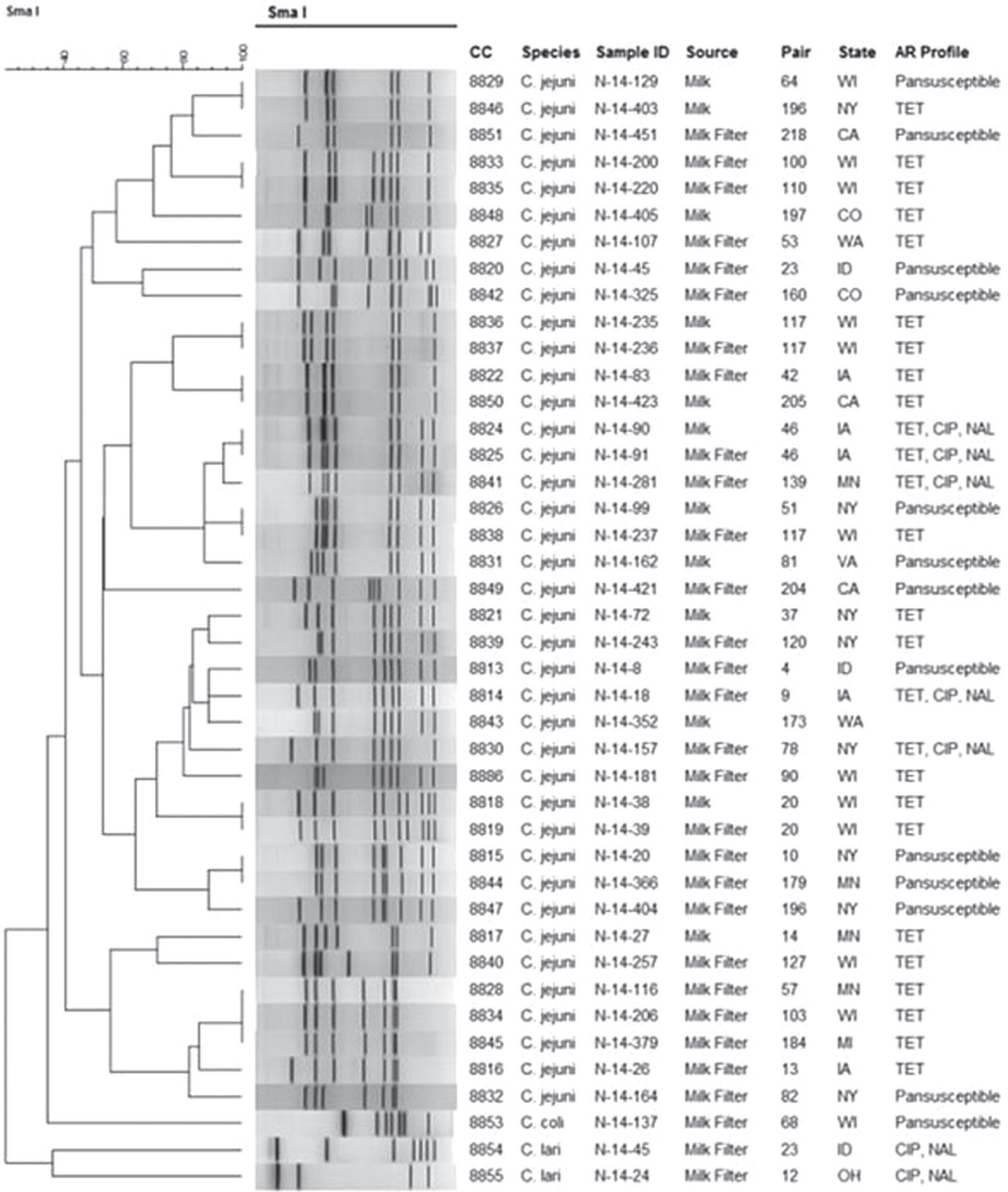

Figure 2. Dendrogram of SmaI-digested pulsed-field gel electrophoresis patterns of Campylobacter spp. isolates (C. jejuni, C. coli, and C. lari) from bulk tank milk and milk filter pairs. TET = tetracycline, CIP = ciprofloxacin, and NAL = nalidixic acid; AR = antimicrobial resistance.

2013). Sampling filters in addition to BTM provides an additional opportunity to identify Campylobacter in milking systems.
Campylobacter spp. isolates were obtained from approximately half of the samples identified as positive via $\mathrm{PCR}$, and, as expected, no isolates were obtained 
from samples that were PCR-negative. Real-time PCR is widely considered to be a more sensitive means of detection than culture techniques, which may produce variable results. Campylobacter spp. are especially challenging to culture in the laboratory and require a microaerophilic environment, as well as supplementation with antimicrobials to inhibit competition from enteric flora. Although an operation-level prevalence of $12.5 \%$ (wp) via culture is similar to previous multistate culture-based surveys of BTM (0.9-12.3\%; Doyle and Roman, 1982; Lovett et al., 1983; McManus and Lanier, 1987; Rohrbach et al., 1992; Jayarao and Henning, 2001), reliance on culture techniques alone may result in a considerable underestimation of Campylobacter spp. prevalence due to the challenges in culture and isolation. Additionally, this survey was a single point-in-time sampling between March and July and may underestimate the actual prevalence of Campylobacter, because only 2 samples (BTM and milk filter) were taken per operation. Previous research indicates that Campylobacter spp. may be shed seasonally in dairy animals, with peaks in the spring in Wisconsin and in spring and fall in the United Kingdom (Stanley et al., 1998; Sato et al., 2004). Previous research into Salmonella also suggests that prevalence in BTM or filters may vary day to day, possibly due to variability in the events that lead to contamination of BTM (Van Kessel et al., 2012; Van Kessel et al., 2013). Previous national surveys based on fecal sampling of dairy cows have shown $90-100 \%$ of dairy operations are positive for Campylobacter spp., but these surveys collected 15-50 samples per operation, which increased the opportunity for detection of Campylobacter (USDA-APHIS, 2011). Therefore, although Campylobacter spp. have been detected in fecal samples from most dairy operations, the detection of Campylobacter in BTM is affected by the amount of fecal contamination in the system at the time of sampling.

The most frequent Campylobacter species associated with dairy animals, as well as human illness, is $C$. jejuni (CDC, 2014), and this was the predominant species isolated in the present study. Of the 49 isolates obtained from BTM and milk filter samples, 45 were C. jejuni; 1 C. jejuni isolate came from a milk filter from which C. lari was also isolated. Therefore, C. jejuni remains the Campylobacter species of greatest concern for the prevention and surveillance of foodborne disease.

An additional concern for foodborne pathogens is the potential for antimicrobial resistance. When we tested the susceptibility of C. jejuni isolates to a panel of common antimicrobials, 12 C. jejuni isolates (31.6\%) were susceptible to all 9 antimicrobials, but $26(68.4 \%)$ were resistant to tetracycline (Table 4). Although BTM and milk filters were not tested for the presence of Campy- lobacter spp. in the NAHMS 2007 study, $62.4 \%$ of $C$. jejuni isolates from fecal samples in 2007 were resistant to tetracycline (USDA-APHIS, 2011). Tetracycline and its derivatives are used on dairy operations in milk replacers and footbaths, and bacterial resistance to this antimicrobial is common (Zwald et al., 2004; USDAAPHIS, 2011). Although campylobacteriosis in humans due to $C$. jejuni is usually self-limiting, erythromycin is the drug of choice when antimicrobial intervention is deemed necessary (Blaser and Engberg, 2008). All of the isolates collected in this survey were susceptible to erythromycin. Fluoroquinolones such as ciprofloxacin may also be used as an alternative to erythromycin, and a recent national survey of antimicrobial resistance in the United States identified resistance to ciprofloxacin in $22 \%$ of human C. jejuni isolates and $8.5 \%$ of dairy fecal isolates (CDC, 2014). We also observed resistance to ciprofloxacin in 5 isolates $(13.2 \%)$ in this study.

The second most common cause of human Campylobacter infection is $C$. coli, although several other species are recognized as human pathogens, including C. lari. One $C$. coli isolate from a milk filter was susceptible to all tested antimicrobials, but we observed resistance to 2 quinolones (ciprofloxacin and nalidixic acid) in $2 C$. lari isolates from milk filter samples. However, C. lari is an infrequent cause of human illness, and it is also considered intrinsically resistant to quinolones (Skirrow and Benjamin, 1980; CDC, 2016). Resistance to common antimicrobials did not appear to be a major attribute of the few $C$. coli and C. lari isolates in this survey; however, BTM may represent an additional source of pathogens, such as C. jejuni, that harbor antimicrobial resistance in the dairy farm environment.

When the $S m a I$ restriction digest patterns were compared, C. coli and C. lari were distinct from the $C$. jejuni isolates, as expected (Figure 2). The C. jejuni strains were genotypically diverse, and clonal strains were geographically dispersed. When comparing the restriction digest patterns of isolates from BTM and filters, we found 9 indistinguishable C. jejuni clonal groups (100\% similarity). Five of these clonal groups comprised isolates from operations in different states. In previous surveys of US dairy farms, Harvey et al. (2005) and Sanad et al. (2011) similarly described this genotypic similarity of isolates collected from different geographic regions. Dissemination across regions has been attributed to many sources, including migratory birds (Sanad et al., 2013; Keller and Shriver, 2014; Ryu et al., 2014). As observed in the present study and previous surveys (Sanad et al., 2011), C. jejuni isolates obtained from US dairy farms are genotypically diverse, and this diversity can extend to the farm level. On 2 occasions, different strains ( $<65 \%$ similarity) were isolated from a single operation. Given the high herd-level 
prevalence on US dairy farms, this farm-level diversity could be due to the shedding of different strains by multiple cows in the milking herd. Alternatively, this could represent co-colonization by multiple strains in a single cow, as described by Nielsen (2002).

When comparisons included both restriction digest patterns and antimicrobial-resistant phenotypes, we observed no consistent association. Harvey et al. (2005) similarly observed that antimicrobial resistance patterns differed within some genotypes. In this study, resistance to tetracycline was dispersed throughout the 4 C. jejuni clades and across geographic regions (Figure 2). Although several indistinguishable $C$. jejuni isolate pairs exhibited similar resistance, in each of 2 pairs of indistinguishable isolates, 1 isolate was resistant to tetracycline and the second was susceptible, possibly attributable to several transmissible plasmid-encoded resistance genes, such as tetO (Taylor, 1986). Based on this and previous studies, antimicrobial resistance is not uncommon in C. jejuni, and phenotypic diversity in antimicrobial resistance may exist within clonal $C$. jejuni groups.

The CDC collects PFGE restriction digest patterns of pathogenic bacterial isolates from humans and other sources in the PulseNet database to identify foodborne outbreaks (Swaminathan et al., 2001). When we compared the PFGE patterns of the Campylobacter spp. isolates with the PulseNet database, 35 of 39 C. jejuni isolates matched pulsetypes isolated from humans in 2015 , of which 7 were from raw milk. Study isolates also matched 22 isolates in the database that were obtained from poultry sources, such as chicken breasts, indicating that Campylobacter strains are not species-specific.

Campylobacter spp. are consistently found in feces of dairy animals in the United States and, in this study, were detected in BTM and milk filters from one-quarter of US dairy operations. As with other recent analyses of Campylobacter isolates and outbreak data, C. jejuni pulsetypes were commonly associated with those from PulseNet isolated from humans. Additionally, $68.4 \%$ of Campylobacter isolates were resistant to at least 1 antimicrobial. The estimated incidence of foodborne outbreaks due to raw milk consumption is over 150 times greater than the incidence due to pasteurized milk consumption (Langer et al., 2012), and Campylobacter spp. are the most common cause of these outbreaks. Therefore, contamination of BTM by Campylobacter spp. can lead to health risks for consumers of raw milk.

\section{ACKNOWLEDGMENTS}

The authors acknowledge the producer participants, the USDA Veterinary Services (Fort Collins, CO) staff for sample collection, Jakeitha Sonnier of USDA-ARS-
EMFSL (Beltsville, MD) for technical assistance, and Shaohua Zhao and Jason Abbott of the FDA-Center for Veterinary Medicine (Laurel, MD) for PulseNet analysis. Mention of trade names or commercial products in this publication is solely for the purpose of providing specific information and does not imply recommendation or endorsement by the USDA.

\section{REFERENCES}

AAP (American Academy of Pediatrics Committee on Infectious Diseases and Committee on Nutrition). 2014. Consumption of raw or unpasteurized milk and milk products by pregnant women and children. Pediatrics 133:175-179.

Bianchini, V., L. Borella, V. Benedetti, A. Parisi, A. Miccolupo, E. Santoro, C. Recordati, and M. Luini. 2014. Prevalence in bulk tank milk and epidemiology of Campylobacter jejuni in dairy herds in Northern Italy. Appl. Environ. Microbiol. 80:1832-1837.

Blaser, M. J., and J. Engberg. 2008. Taxonomy of the family Campylobacteraceae. Pages 99-121 in Campylobacter. 3rd ed. I. Namchamkin, C. Szymanski, and M. Blaser, ed. ASM, Washington, DC.

CDC (Centers for Disease Control and Prevention). 2014. National Antimicrobial Resistance Monitoring System. NARMS Integrated Report 2012-2013. CDC, Atlanta, GA.

CDC (Centers for Disease Control and Prevention). 2016. Food Safety Report 2015. CDC, Atlanta, GA. Accessed Jan. 11, 2017. http:// www.cdc.gov/foodnet/pdfs/foodnet-mmwr-progress-508-final.pdf.

Doyle, M. P., and D. J. Roman. 1982. Prevalence and survival of Campylobacter jejuni in unpasteurized milk. Appl. Environ. Microbiol. 44:1154-1158.

Giacometti, F., A. Serraino, G. Finazzi, P. Daminelli, M. N. Losio, P. Bonilauri, N. Arrigoni, A. Garigliani, R. Mattioli, S. Alonso, S. Piva, D. Florio, R. Riu, and R. G. Zanoni. 2012. Foodborne pathogens in in-line milk filters and associated on-farm risk factors in dairy farms authorized to produce and sell raw milk in northern Italy. J. Food Prot. 75:1263-1269.

Häkkinen, M., and M. L. Hänninen. 2009. Shedding of Campylobacter spp. in Finnish cattle on dairy farms. J. Appl. Microbiol. 107:898905.

Halbert, L. W., J. B. Kaneene, P. L. Ruegg, L. D. Warnick, S. J. Wells, L. S. Mansfield, C. P. Fossler, A. M. Campbell, and A. M. GeigerZwald. 2006. Evaluation of antimicrobial susceptibility patterns in Campylobacter spp. isolated from dairy cattle and farms managed organically and conventionally in the midwestern and northeastern United States. J. Am. Vet. Med. Assoc. 228:1074-1081.

Harvey, R. B., M. E. Hume, R. E. Droleskey, T. S. Edrington, C. L. Sheffield, T. R. Callaway, R. L. Ziprin, H. M. Scott, R. C. Anderson, and D. J. Nisbet. 2005. Further characterization of Campylobacter isolated from U.S. dairy cows. Foodborne Pathog. Dis. $2: 182-187$.

Jayarao, B. M., S. C. Donaldson, B. A. Straley, A. A. Sawant, N. V. Hegde, and J. L. Brown. 2006. A survey of foodborne pathogens in bulk tank milk and raw milk consumption among farm families in Pennsylvania. J. Dairy Sci. 89:2451-2458.

Jayarao, B. M., and D. R. Henning. 2001. Prevalence of foodborne pathogens in bulk tank milk. J. Dairy Sci. 84:2157-2162.

Keller, J. I., and W. G. Shriver. 2014. Prevalence of three campylobacter species, C. jejuni, $C$. coli, and C. lari, using multilocus sequence typing in wild birds of the Mid-Atlantic region, USA. J. Wildl. Dis. 50:31-41.

Langer, A. J., T. Ayers, J. Grass, M. Lynch, F. J. Angulo, and B. E. Mahon. 2012. Nonpasteurized dairy products, disease outbreaks, and state laws-United States, 1993-2006. Emerg. Infect. Dis. 18:385-391.

Latorre, A. A., A. K. Pradhan, J. A. Van Kessel, J. S. Karns, K. J. Boor, D. H. Rice, K. J. Mangione, Y. T. Gröhn, and Y. H. Schukken. 2011. Quantitative risk assessment of listeriosis due to consumption of raw milk. J. Food Prot. 74:1268-1281. 
Leone, P., P. Cremonesi, and A. Stella. 2010. Molecular-based identification of pathogens (Staphylococcus aureus, Bacillus cereus, Campylobacter spp., and Streptococcus agalactiae) in raw milk and milk filter residuals. Renc. Rech. Ruminants. 17:108.

Lovett, J., D. W. Francis, and J. M. Hunt. 1983. Isolation of Campylobacter jejuni from raw milk. Appl. Environ. Microbiol. 46:459-462.

McManus, C., and J. M. Lanier. 1987. Salmonella, Campylobacter jejuni, and Yersinia enterocolitica in raw milk. J. Food Prot. 50:51-55.

Morgan, G., P. Chadwick, K. P. Lander, and K. P. W. Gill. 1985. Campylobacter jejuni mastitis in a cow: A zoonosis-related incident. Vet. Rec. 116:111.

Mungai, E. A., C. B. Behravesh, and L. H. Gould. 2015. Increased outbreaks associated with nonpasteurized milk, United States, 2007-2012. Emerg. Infect. Dis. 21:119-122.

NCSL (National Conference of State Legislatures). 2016. State milk laws. Accessed Dec. 7, 2012. http://www.ncsl.org/research/ agriculture-and-rural-development/raw-milk-2012.aspx.

Nielsen, E. M. 2002. Occurrence and strain diversity of thermophilic campylobacters in cattle of different age groups in dairy herds. Lett. Appl. Microbiol. 35:85-89.

Oliver, S. P., B. M. Jayarao, and R. A. Almeida. 2005. Foodborne pathogens in milk and the dairy farm environment: Food safety and public health implications. Foodborne Pathog. Dis. 2:115-129.

Orr, K. E., N. F. Lightfoot, P. R. Sisson, B. A. Harkis, J. L. Tweddle, P. Boyd, A. Carroll, C. J. Jackson, D. R. Wareing, and R. Freeman. 1995. Direct milk excretion of Campylobacter jejuni in a dairy cow causing cases of human enteritis. Epidemiol. Infect. 114:15-24.

Rapp, D., C. M. Ross, E. J. Pleydell, and R. W. Muirhead. 2012. Differences in the fecal concentrations and genetic diversities of Campylobacter jejuni populations among individual cows in two dairy herds. Appl. Environ. Microbiol. 78:7564-7571.

Ribot, E. M., C. Fitzgerald, K. Kubota, B. Swaminathan, and T. J. Barrett. 2001. Rapid pulsed-field gel electrophoresis protocol for subtyping of Campylobacter jejuni. J. Clin. Microbiol. 39:18891894.

Rohrbach, B. W., F. A. Draughon, and P. M. Davidson. 1992. Prevalence of Listeria monocytogenes, Campylobacter jejuni, Yersinia enterocolitica, and Salmonella in bulk tank milk: Risk factors and risk of human exposure. J. Food Prot. 55:93-97.

Ryu, H., K. Grond, B. Verheijen, M. Elk, D. M. Buehler, and J. W. Santo Domingo. 2014. Intestinal microbiota and species diversity of Campylobacter and Helicobacter spp. in migrating shorebirds in Delaware Bay. Appl. Environ. Microbiol. 80:1838-1847.

Sanad, Y. M., G. Closs, A. Kumar, J. T. LeJeune, and G. Rajashekara. 2013. Molecular epidemiology and public health relevance of Campylobacter isolated from dairy cattle and European starlings in Ohio, USA. Foodborne Pathog. Dis. 10:229-236.

Sanad, Y. M., I. I. Kassem, M. Abley, W. Gebreyes, J. T. LeJeune, and G. Rajashekara. 2011. Genotypic and phenotypic properties of cattle-associated Campylobacter and their implications to public health in the USA. PLoS One 6:e25778.

Sato, K., P. C. Bartlett, J. B. Kaneene, and F. P. Downes. 2004. Comparison of prevalence and antimicrobial susceptibilities of Campylobacter spp. isolates from organic and conventional dairy herds in Wisconsin. Appl. Environ. Microbiol. 70:1442-1447.

Schleif, R. F., and P. C. Wensink. 1981. Practical Methods in Molecular Biology. Springer-Verlag, New York.
Schwarz, S., P. Silley, S. Simjee, N. Woodford, E. van Duijkeren, A. P. Johnson, and W. Gaastra. 2010. Editorial: Assessing the antimicrobial susceptibility of bacteria obtained from animals. J. Antimicrob. Chemother. 65:601-604

Serraino, A., D. Florio, F. Giacometti, S. Piva, D. Mion, and R. G. Zanoni. 2013. Presence of Campylobacter and Arcobacter species in in-line milk filters of farms authorized to produce and sell raw milk and of a water buffalo dairy farm in Italy. J. Dairy Sci. 96:2801-2807.

Skirrow, M. B., and J. Benjamin. 1980. Differentiation of enteropathogenic Campylobacter. J. Clin. Pathol. 33:1122.

Stanley, K. N., J. Wallace, J. E. Currie, P. J. Diggle, and K. Jones. 1998. The seasonal variation of thermophilic campylobacters in beef cattle, dairy cattle and calves. J. Appl. Microbiol. 85:472-480.

Swaminathan, B., T. J. Barrett, S. B. Hunter, and R. V. Tauxe. 2001. PulseNet: The molecular subtyping network for foodborne bacterial disease surveillance, United States. Emerg. Infect. Dis. 7:382-389.

Taylor, D. E. 1986. Plasmid-mediated tetracycline resistance in Campylobacter jejuni: Expression in Escherichia coli and identification of homology with streptococcal class M determinant. J. Bacteriol. 165:1037-1039.

Taylor, E. V., K. M. Herman, E. C. Ailes, C. Fitzgerald, J. S. Yoder, B. E. Mahon, and R. V. Tauxe. 2013. Common source outbreaks of Campylobacter infection in the USA, 1997-2008. Epidemiol. Infect. 141:987-996.

Tyson, G. H., H. Tate, J. Abbott, T. Tran, C. Kabera, E. Crarey, S. Young, P. F. McDermott, G. Sprague, M. Campbell, O. Adeyemo, J. Browne-Silva, M. Myers, S. Thitaram, and S. Zhao. 2016. Molecular subtyping and source attribution of Campylobacter isolated from food animals. J. Food Prot. 79:1891-1897.

USDA-APHIS (USDA Animal and Plant Health Inspection Service). 2011. Salmonella, Listeria, and Campylobacter on U.S. Dairy Operations, 1996-2007. USDA-APHIS-VS, CEAH, Fort Collins, CO.

Van Kessel, J. A., J. S. Karns, J. E. Lombard, and C. A. Kopral. 2011. Prevalence of Salmonella enterica, Listeria monocytogenes, and Escherichia coli virulence factors in bulk tank milk and in-line filters from U.S. dairies. J. Food Prot. 74:759-768.

Van Kessel, J. A., J. S. Karns, D. R. Wolfgang, and E. Hovingh. 2013. Regional distribution of two dairy-associated Salmonella enterica serotypes. Foodborne Pathog. Dis. 10:448-452.

Van Kessel, J. S., J. S. Karns, D. R. Wolfgang, E. Hovingh, B. M. Jayarao, C. P. Van Tassell, and Y. H. Schukken. 2008. Environmental sampling to predict fecal prevalence of Salmonella in an intensively monitored dairy herd. J. Food Prot. 71:1967-1973.

Van Kessel, J. A., J. S. Karns, D. R. Wolfgang, E. Hovingh, and Y. H. Schukken. 2012. Dynamics of Salmonella serotype shifts in an endemically infected dairy herd. Foodborne Pathog. Dis. 9:319-324.

Wang, G., C. G. Clark, T. M. Taylor, C. Pucknell, C. Barton, L. Price, D. L. Woodward, and F. G. Rodgers. 2002. Colony Multiplex PCR assay for identification and differentiation of Campylobacter jejuni, C. coli, C. lari, C. upsaliensis, and C. fetus ssp. fetus. J. Clin. Microbiol. 40:4744-4747.

Zwald, A. G., P. L. Ruegg, J. B. Kaneene, L. D. Warnick, S. J. Wells, C. Fossler, and L. W. Halbert. 2004. Management practices and reported antimicrobial usage on conventional and organic dairy farms. J. Dairy Sci. 87:191-201. 